\title{
ON INHIBITING RUNAWAY IN CATALYTIC REACTORS*
}

\author{
DONALD S. COHEN $\dagger$ AND BERNARD J. MATKOWSKY
}

\begin{abstract}
We consider the problem of heat and mass transfer in porous catalyst pellets. Both the steady and time dependent operating characteristics are studied. Accurate approximate equations are derived from the basic governing equations of motion. A nonlinear stability analysis is employed to account for the observation that under certain conditions reactions on catalyst pellets can pass transiently stably into a region which would correspond to instability in the steady state. One consequence of our analysis is a possible control mechanism which inhibits temperature runaway by extending the stable operating characteristics desirable in modern reactors.
\end{abstract}

1. Introduction. It is well-known that under certain conditions multiple steady states occur when exothermic reactions are carried out in a porous catalyst pellet. For such a reaction inside a tubular reactor a typical response diagram (the behavior of the amplitude of the temperature $\|v\|$ as one of the parameters, the Damkohler number $D$, varies) is given in Fig. 1. The upper and lower branches of the curve are stable, while the middle branch is unstable. If $D$ is increased past $D_{A}$, or if the temperature is increased above the middle branch, the temperature undergoes a jump (an ignition) to the upper branch. This is sometimes referred to as temperature runaway. Similarly, if $D$ is decreased below $D_{B}$ or if the temperature is decreased below the middle branch, the temperature undergoes a jump (an extinction) to the lower branch. Thus the system exhibits a hysteresis effect.

In certain types of reactions on the pellets, where the upper temperature branch represents an undesirable state corresponding to interphase mass transfer, temperature runaway is to be avoided. That is, even though the upper branch is stable in the usual sense, it may be undesirable in some applications to have the reactor operate at such high temperatures. In these cases it would be desirable to prevent runaway.

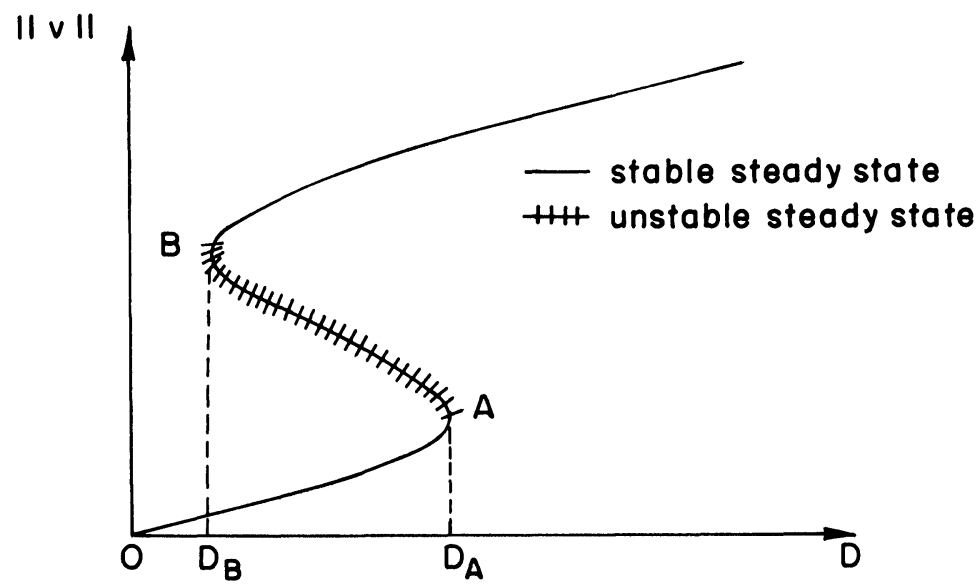

FIG. 1

* Received by the editors April 14, 1976, and in revised form November 2, 1977. This work was supported in part by the U.S. Army Research Office under Grants DAHC04-75-C-0009, DAAG 29-77-G0222, the National Science Foundation under MPS 75-05414, MCS 77-25660, and by the U.S. Energy Research and Development Adminstration.

$\dagger$ Department of Applied Mathematics, California Institute of Technolgy, Pasadena, California 91125.

¥Department of Engineering Sciences and Applied Mathematics, Northwestern University, Evanston, Illinois, 60201. 
Computer studies by C. McGreavy and J. M. Thornton [1] have shown that under external temperature fluctuations (of sufficiently high frequency) it is possible to inhibit runaway.

The main goal of our analysis is to explain and account for the numerical results of McGreavy and Thornton from the equations governing the process and to give analytical conditions for the occurrence of the observed phenomena. Specifically we show analytically that if the frequency of the applied oscillation exceeds a critical value which we explicitly compute in terms of its dependence on the parameters of the problem, then a stable limit cycle can be set up in the neighborhoods of the points $A$ and $B$. Then, if $D$ exceeds $D_{A}$ at a certain time or if the temperature exceeds the middle branch at a certain time, the system, instead of jumping from $A$ to the upper branch will jump onto the limit cycle which remains in a neighborhood of point $A$. Similar remarks hold for point $B$. Thus a consequence of this analysis is a control mechanism for inhibiting temperature runaway by extending the region of low temperature stability. Our analysis is carried out on approximate equations presented by McGreavy and Thornton [1], [2]. The relationship of these approximation equations to the basic equations of motion is discussed in Appendix A.

2. The catalytic reactor. The mathematical problem of heat and mass transfer in a spherically shaped catalyst pellet undergoing Arrhenius kinetics can be written in dimensionless form as

$$
\begin{aligned}
& k_{1} C_{t}=C_{x x}+\frac{2}{x} C_{x}-D C e^{-1 / T}, \quad 0<x<1, \quad t>0, \\
& k_{2} T_{t}=T_{x x}+\frac{2}{x} T_{x}+D B C e^{-1 / T}, \quad 0<x<1, \quad t>0, \\
& C_{x}(0, t)=0, \quad t \geqq 0, \\
& T_{x}(0, t)=0, \quad t \geqq 0, \\
& C_{x}(1, t)=S[\bar{C}(t)-C(1, t)], \quad t \geqq 0, \\
& T_{x}(1, t)=N[\bar{T}(t)-T(1, t)], \quad t \geqq 0, \\
& C(x, 0)=\phi(x), \quad 0 \leqq x \leqq 1, \\
& T(x, 0)=\psi(x), \quad 0 \leqq x \leqq 1 .
\end{aligned}
$$

Here $C(x, t)$ is the concentration. $T(x, t)$ is the temperature, the functions $\bar{C}(t)$ and $\bar{T}(t)$ are known functions representing the temporal variations of the surrounding concentration and temperature fields respectively, and the other quantities are prescribed physical constants.

When $\bar{C}(t) \equiv$ const., C. McGreavy and J. M. Thornton [1], [2] present arguments to show for all practical values of the physical parameters, the temperature field is approximately homogeneous in space (i.e., $T(x, t) \sim v(t)$ for all $x$ in $0 \leqq x \leqq 1)$ and $v(t)$ satisfies the equation

$$
k_{2} v^{\prime}=\bar{T}(t)-g(v)
$$

where $g(v)$ is of the form illustrated in Fig. 2. In Appendix A we show that a similar approximation can be derived for fluctuating temperature and concentration fields. In this section we shall study (2.9) when there are small sinusoidal fluctuations in the external temperature; namely $\bar{T}(t)=\lambda+\beta \sin \omega t$. 


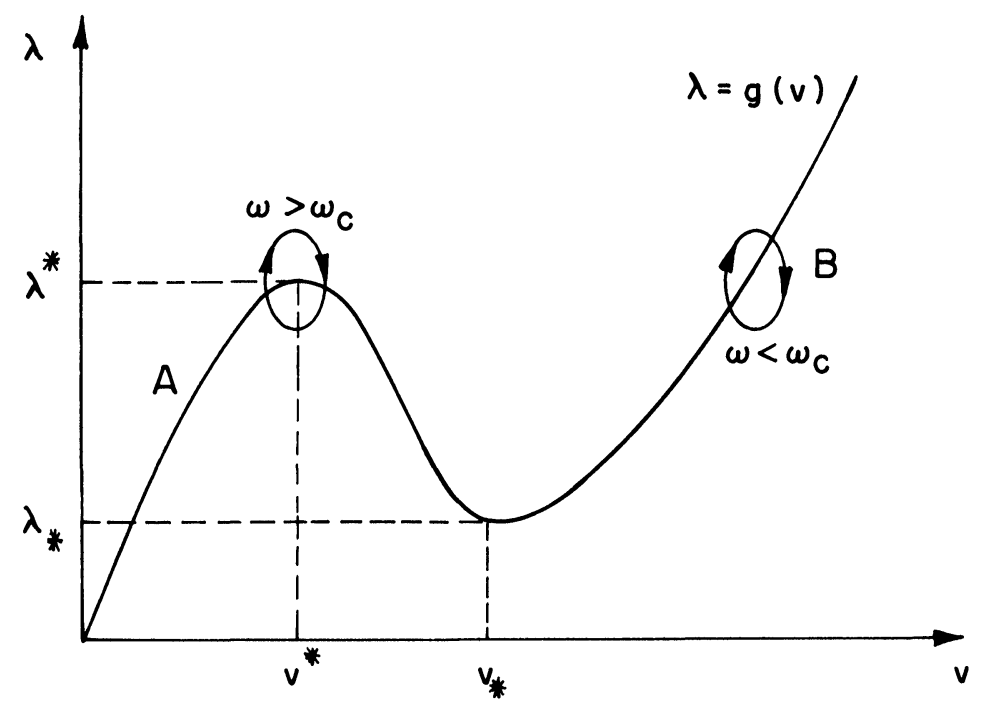

FIG. 2

Equation (2.9) is of the form

$$
v^{\prime}=\Lambda(t)-g(v)
$$

When the ambient temperature is constant for a reactor or when the surrounding fluid temperature is constant for a porous catalyst pellet, $\Lambda(t)$ is constant, say $\Lambda(t) \equiv \lambda$, so that

$$
v^{\prime}=\lambda-g(v)
$$

Steady state solutions are given by solutions of $\lambda=g(v)$, and for nonlinearities of the form illustrated in Fig. 2 we see immediately that there exists one steady state solution for $\lambda<\lambda_{*}$ or $\lambda>\lambda^{*}$, two steady state solutions for $\lambda=\lambda_{*}$ or $\lambda=\lambda^{*}$, and three steady state solutions for $\lambda_{*}<\lambda<\lambda^{*}$.

To examine the stability of these solutions we note that (2.11) implies that $v^{\prime}$ is greater than (less than) zero according as $\lambda-g(v)$ is greater than (less than) zero. Thus, the upper and lower solution branches are stable while the middle branch is unstable., (See Fig. 2.) Hence, $\lambda=\lambda^{*}\left(\lambda_{*}\right)$ is the critical value at which temperature runaway occurs, or equivalently, $v=v^{*}\left(v_{*}\right)$ is the stability boundary.

3. A control mechanism. We shall now show that if the external temperature $\Lambda(t)$ is made to fluctuate in time, then runaway can be inhibited by setting up a stable limit cycle near $\left(\lambda_{*}, v_{*}\right)$ or $\left(\lambda^{*}, v^{*}\right)$. Thus, we consider the problem (2.10) with $\Lambda(t)=\lambda+\beta \sin \omega t$, where $0<\beta \ll 1$, subject to the initial condition $v(0)=v_{0}+\beta V$, where $v_{0}$, for a given $\lambda$, is a steady state solution given by

$$
\lambda=g\left(v_{0}\right),
$$

and where $g(v)$ is illustrated in Fig. 2. First, we seek an asymptotic expansion (the outer expansion) of the solution of the form

$$
v(t) \sim \sum_{n=0}^{\infty} v_{n}(t) \beta^{n}
$$


where $v_{0}(t) \equiv v_{0}$ given by (3.1), and the $v_{n}(t)$ are uniformly bounded in $t$. Inserting (3.2) into (2.10) with $\Lambda(t)=\lambda+\beta \sin \omega t$ and equating like powers of $\beta$, we find that to order $\beta^{2}$ we obtain

$$
\begin{array}{cc}
v_{1}^{\prime}+g^{\prime}\left(v_{0}\right) v_{1}=\sin \omega t, & v_{1}(0)=V, \\
v_{2}^{\prime}+g^{\prime}\left(v_{0}\right) v_{2}=-\frac{1}{2} g^{\prime \prime}\left(v_{0}\right) v_{1}^{2}, & v_{2}(0)=0 .
\end{array}
$$

The solution of (3.3) is given by

$$
v_{1}(t)=\left[V+\frac{\omega}{\omega^{2}+\gamma^{2}}\right] e^{-\gamma t}+\frac{1}{\omega^{2}+\gamma^{2}}[\gamma \sin \omega t-\omega \cos \omega t],
$$

if

$$
\gamma=g^{\prime}\left(v_{0}\right) \neq 0 .
$$

Clearly, if $\gamma>0$ (corresponding to the branches $v<v^{*}$ and $v>v_{*}$ in Fig. 2), then as $t \rightarrow \infty, v_{1}(t)$ becomes oscillatory with amplitude $1 / \sqrt{\omega^{2}+\gamma^{2}}$ and frequency $\omega$ for any applied frequency. If $\gamma=0$, the solutions of (3.3) and (3.4) are given by

$$
\begin{aligned}
& v_{1}(t)=V+\frac{1}{\omega}-\frac{1}{\omega} \cos \omega t \\
& v_{2}(t)=-\alpha\left[\left(V+\frac{1}{\omega}\right)^{2}+\frac{1}{2 \omega^{2}}\right] t+\frac{2 \alpha}{\omega^{2}}\left(V+\frac{1}{\omega}\right) \sin \omega t-\frac{\alpha}{4 \omega^{3}} \sin 2 \omega t,
\end{aligned}
$$

where

$$
\alpha=\frac{1}{2} g^{\prime \prime}\left(v_{0}\right) \text {. }
$$

Assuming that $\alpha \neq 0$, we observe that the asymptotic expansion breaks down in the neighborhood of the points $\lambda=\lambda_{*}$ and $\lambda=\lambda^{*}$ since $v_{2}(t)$ with $\gamma=0$ grows in $t$. We refer to these neighborhoods as boundary layers. Thus, away from these boundary layers the asymptotic expansion of the solution is given by (3.2) with leading term $v_{1}$ given by (3.5). It describes a forced stable oscillation of amplitude $\beta / \sqrt{\omega^{2}+\gamma^{2}}$ and frequency $\omega$ for any applied frequency $\omega$. This solution is not valid in the boundary layers and new expansions must be derived in these regions. These are now constructed.

In the boundary layer near $\lambda=\lambda_{*}$ we introduce the stretched variable

$$
\tilde{\lambda}=\left(\lambda-\lambda_{*}\right) / \beta^{2},
$$

and seek an asymptotic expansion (the inner expansion) in the form

$$
v-v_{*} \sim \sum_{n=1} u_{n}(t, \tau) \beta^{n}
$$

where the $u_{n}(t, \tau)$ are uniformly bounded in time, and the "slow time" $\tau$ is defined as

$$
\tau=\beta t \text {. }
$$

(For the layer near $\lambda=\lambda^{*}$ we would proceed similarly with the stretched variable $\tilde{\lambda}=\left(\lambda^{*}-\lambda\right) / \beta^{2}$.) Upon substituting (3.10)-(3.12) into (2.10) with $\Lambda(t)=\lambda+\beta \sin \omega t$ and equating like powers of $\beta$, we find that to order $O\left(\beta^{2}\right)$ we obtain

$$
\begin{gathered}
\frac{\partial u_{1}}{\partial t}=\sin \omega t, \quad u_{1}(0,0)=V, \\
\frac{\partial u_{2}}{\partial t}=\tilde{\lambda}-\alpha u_{1}^{2}-\frac{\partial u_{1}}{\partial \tau}, \quad u_{2}(0,0)=0 .
\end{gathered}
$$


The solution of the equation in (3.13) is given by

$$
u_{1}(t, \tau)=-\frac{1}{\omega} \cos \omega t+a(\tau),
$$

where the unknown function $a(\tau)$ is to be determined at a later stage of the perturbation procedure. The initial condition in (3.13) implies that

$$
a(0)=V+1 / \omega
$$

Using (3.15), we find that (3.14) becomes

$$
\frac{\partial u_{2}}{\partial t}=\left[\tilde{\lambda}-\frac{\alpha}{2 \omega^{2}}-\alpha a^{2}-\frac{d a}{d \tau}\right]+\frac{2 \alpha a}{\omega} \cos \omega t-\frac{\alpha}{2 \omega^{2}} \cos 2 \omega t
$$

Now, the solution $u_{2}$ will be bounded if and only if

$$
\frac{d a}{d \tau}+\alpha a^{2}=\tilde{\lambda}-\frac{\alpha}{2 \omega^{2}} \text {. }
$$

The solution of (3.18) is given by

$$
a(\tau)=p\left[\frac{1-k e^{-2 \alpha p \tau}}{1+k e^{-2 \alpha p \tau}}\right],
$$

where

$$
p=\sqrt{\tilde{\lambda} / \alpha-1 /\left(2 \omega^{2}\right)}
$$

and where $k$ is determined from the initial condition (3.16) as a solution of

$$
V+\frac{1}{\omega}=p\left[\frac{1+k}{1+k}\right]
$$

Note that $a(\tau) \rightarrow p$ as $\tau \rightarrow \infty$ independent of the initial conditions. Clearly, the requirements that $a(\tau)$ be bounded for all $\tau$ and that $a(\tau)$ and $\omega$ be real imply that

$$
\frac{\tilde{\lambda}}{\alpha}>\frac{1}{2 \omega^{2}}>0
$$

from which it immediately follows that $\lambda_{*}<\lambda<\lambda^{*}$, and

$$
\omega>\omega_{\mathrm{c}} \equiv \sqrt{\frac{\alpha}{2 \tilde{\lambda}}}=\beta \sqrt{\frac{\alpha}{2\left(\lambda-\lambda_{*}\right)}} .
$$

Therefore, near $\lambda=\lambda_{*}$ the applied frequency $\omega$ must exceed the critical frequency $\omega_{\mathrm{c}}$ defined by (3.23) for stable oscillation to occur, and to first order in $\beta$ we see from (3.11), (3.15), and (3.19) that this stable oscillation is given in the limit as $t \rightarrow \infty$ by

$$
v \rightarrow v_{*}+\beta[p-(1 / \omega) \cos \omega t]
$$

Before studying the implications of our analysis we shall complete our derivation by showing that the outer expansion (3.2) and the inner expansion (3.11) match to the order to which we have carried out the derivation, namely to order $O(\beta)$. In the usual way we postulate that both the inner and outer expansions are valid in some common region where they overlap, and this overlap region is given in terms of the intermediate variable $\lambda_{\eta}=\left(\lambda-\lambda_{*}\right) / \eta(\beta)$, where $\beta^{2}<\eta(\beta)<1$. Thus, by writing both inner and outer expansions in terms of the variable $\lambda_{\eta}$, we should find that they agree to 
order $O(\beta)$ as $\beta \rightarrow 0$. Note that $\lambda=\lambda_{*}+\eta(\beta) \lambda_{\eta} \rightarrow \lambda_{*}$ as $\beta \rightarrow 0$. Then, from (3.13), (3.15) and (3.16) we see that as $\beta \rightarrow 0$, the inner solution has the property that

$$
v \rightarrow v_{*}+\beta\left[-\frac{1}{\omega} \cos \omega t+V+\frac{1}{\omega}\right]
$$

and from (3.2) and (3.5) we see that as $\omega \rightarrow 0$ (implying $\lambda \rightarrow \lambda_{*}, v_{0} \rightarrow v_{*}$, and $\gamma \rightarrow 0$ ), the outer solution approaches the same limit. Therefore, the inner and outer expansions match.

We have given the derivation for $\lambda$ near $\lambda_{*}$. Clearly, a similar analysis applied near $\lambda=\lambda^{*}$. Thus, we have found for (2.10) with $\Lambda(t)=\lambda^{*}+\beta \sin \omega t(0<\beta \ll 1)$, as $t \rightarrow \infty$, the solution $v(t)$ approaches a stable oscillation given by

$$
v(t) \rightarrow v^{*}+\beta\left[-\frac{1}{\omega} \cos \omega t+P\right], \quad \text { where } P=\sqrt{\frac{2\left(\lambda-\lambda^{*}\right)}{g^{\prime \prime}\left(v^{*}\right)}-\frac{1}{2 \omega^{2}}}
$$

provided that the applied frequency $\omega$ satisfies the condition that

$$
\omega>\beta \sqrt{\frac{1}{2}\left|\frac{\alpha}{\lambda-\lambda_{*}}\right|} \equiv \omega_{\mathrm{c}}
$$

Thus, as $t \rightarrow \infty$,

$$
\frac{\omega^{2}}{\beta^{2}}\left(v(t)-v^{*}-\beta P\right)^{2}+\left(\Lambda(t)-\lambda^{*}\right)^{2} \rightarrow 1
$$

This describes an ellipse which represents a limit cycle about the point $\left(v^{*}+\beta P, \lambda^{*}\right)$. Thus we see to what extent the temperature $v^{*}$ can be exceeded without temperature runaway. If the frequency $\omega$ falls below the critical value $\omega_{\mathrm{c}}$, then $a(\tau)$ becomes unbounded so that the lower temperature branch ceases to be an attractor, and temperature runaway occurs. The ensuing solution can then not be followed by our perturbation technique. The numerical calculations of McGreavy and Thornton [1] show that in this case the upper temperature branch becomes the attractor, and the subsequent temperature is a limit cycle around this branch. This is illustrated schematically in Fig. 2, but not drawn to scale for any particular set of numerical values. From the point of view of inhibiting temperature runaway when operating near $\lambda_{*}$ or $\lambda^{*}$ we can see that the application of a sinusoidal "forcing function" $\beta \sin \omega t$ with a frequency in excess of the critical frequency $\omega_{c}$ is a possible control device for stabilization.

Appendix A. Discussion of approximate equations. Equation (2.9) is employed in the analysis of McGreavy and Thornton [1], [2], who considered the case of fluctuating temperature but constant concentration. Expanding on the physical reasoning set forth by McGreavy and Thornton, we show that for temperature and concentration both fluctuating in time, a similar approximation results. When the dominant physical mechanisms are chemical reaction and molecular diffusion, as is the case in most problems involving reactors or reactions in a catalyst pellet, the basic equations for a single reaction are of the form

$$
k_{1} u_{t}=\Delta u-f(u, v)
$$$$
\mathbf{x} \in D, \quad t>0,
$$

$$
k_{2} v_{t}=L \Delta v+b f(u, v)
$$$$
\mathbf{x} \in D, \quad t>0 \text {, }
$$ 
subject to the boundary and initial conditions

$$
\frac{\partial u}{\partial n}=S\left(u_{0}-u\right), \quad \mathbf{x} \in \partial D, \quad t \geqq 0,
$$

$$
\frac{\partial v}{\partial n}=N\left(v_{0}-v\right), \quad \mathbf{x} \in \partial D, \quad t \geqq 0,
$$

$$
\begin{array}{ll}
u(\mathbf{x}, 0)=\phi(x), & \mathbf{x} \in D, \\
v(\mathbf{x}, 0)=\psi(x), & \mathbf{x} \in D .
\end{array}
$$

Here $k_{1}, k_{2}, L, b, S$, and $N$ are known dimensionless constants (see R. Aris [3] for an excellent presentation of the basic equations), $D$ is the basic domain with boundary $\partial D, \partial / \partial n$ denotes differentiation in the direction of the outward pointing normal, and $f(u, v)$ is the rate (or reaction) fraction specifying the chemical kinetics. In most applications $f(u, v)$ is taken as the Arrhenius rate function which is of the form

$$
f(u, v)=P_{n}(u) e^{-k / v}
$$

where $P_{n}(u)$ denotes a polynomial in the concentration $u$ and $v$ is the temperature.

For all practical values of the physical parameters it has been found [2] that the catalyst pellet is essentially isothermal (that is, the catalyst is at a spatially uniform temperature) with the temperature change taking place almost exclusively across a thin fluid film surrounding the catalyst. The temperature $v \equiv v(t)$ in this film is equal to the surface temperature of the pellet. In the usual manner, a heat balance across the film accounts for the rate of change of temperature by heat flux proportional to the difference between fluid $v_{0}$ and pellet $v$ temperature and generation of heat due to chemical reaction. In dimensionless form this heat balance becomes

$$
\frac{d v}{d t}=\alpha_{1}\left(v_{0}-v\right)+\alpha_{2} \int_{D} f(u, v) d \mathbf{x}
$$

where $\alpha_{1}$ and $\alpha_{2}$ are constants.

In a porous catalyst pellet the steady state distribution of concentration is very rapidly reached, and thus, to a first approximation (A.1) becomes

$$
0=\Delta u-f(u, v)
$$

Upon using (A.9), we can write (A.8) as

$$
\frac{d v}{d t}=\alpha_{1}\left(v_{0}-v\right)+\alpha_{2} \int_{D} \Delta u d \mathbf{x}
$$

Now, use the divergence theorem (or Green's theorem) to reduce the volume integral in (A.10) to a surface integral. We obtain

$$
\frac{d v}{d t}=\alpha_{1}\left(v_{0}-v\right)+\alpha_{2} \int_{\partial D} \frac{\partial u}{\partial n} d \sigma .
$$

Upon using the boundary condition (A.3), we find that (A.11) becomes

$$
\frac{d v}{d t}=\alpha_{1}\left(v_{0}-v\right)+\alpha_{2} S \int_{\partial D}\left(u_{0}-u\right) d \sigma
$$


Now, for a spherical pellet of radius $R, u$ is known to be spatially constant on the boundary, and (A.12) becomes

$$
\frac{d v}{d t}=\alpha_{1}\left(v_{0}-v\right)+4 \pi R^{2} \alpha_{2} S\left(u_{0}-\left.u\right|_{\mathbf{x} \in \partial D}\right)
$$

With the pellet isothermal (A.9) can be viewed as an equation for the concentration $u$ (with $v$ as a parameter). Once $u$ is found it is evaluated on the boundary and substituted into (A.13) which then reduces to the form of (2.10). Therefore, we see that the pellet surface temperature and temperature in the fluid film for a porous catalyst pellet is given by the solution of (2.10).

\section{REFERENCES}

[1] C. MCGreavy AND J. M. Thornton, Stability studies of single catalyst particles, Chem. Engng. J., 1 (1970), pp. 296-301.

[2] — Generalized criteria for the stability of catalytic reactors, Canad. J. Chem. Engng., 48 (1970), pp. 187-191.

[3] R. ARIS, The Mathematical Theory of Diffusion and Reaction in Permeable Catalysts, 2 vols., Oxford University Press, London, 1975. 